\title{
Simulador para medição de paralelismo em algoritmos de escalonamento para Replicação Máquina de Estados Paralela
}

\author{
João Gabriel Trombeta ${ }^{1}$, Odorico Machado Mendizabal ${ }^{1}$ \\ ${ }^{1}$ Departamento de Informática e Estatística - Universidade Federal de Santa Catarina \\ Caixa Postal 476 - 88040-900 - Florianópolis - SC - Brasil \\ joao.gabriel.trombeta@grad.ufsc.br, odorico.mendizabal@ufsc.br
}

\begin{abstract}
Resumo. Ao desenvolver um novo algoritmo de escalonamento de requisições para Replicação Máquina de Estados Paralela, é difícil mensurar seu grau de paralelismo sob diferentes cargas de trabalho e configurações, ou comparálo com técnicas existentes. Neste trabalho é proposto um simulador que abstrai custos de uma implementação real, para que sejam analisados possíveis ganhos de desempenho decorrentes exclusivamente das estratégias de escalonamento.
\end{abstract}

\section{Introdução}

Replicação Máquina de Estado (RME) [Lamport 1978, Schneider 1990] é uma técnica utilizada para garantir tolerância a falhas em sistemas distribuídos, ao mesmo tempo que garante consistência forte. Em RME, todas as réplicas partem do mesmo estado inicial e executam as mesmas requisições na mesma ordem, garantindo assim que todas atravessem os mesmos estados. A execução sequencial representa uma limitação no desempenho do sistema, abrindo espaço para implementações de RME Paralela [Kotla and Dahlin 2004], que visam extrair paralelismo na execução de requisições, sem comprometer a consistência. Com o surgimento dessa técnica, estratégias para balancear a execução de requisições entregues às réplicas ganham importância.

Diversas técnicas foram propostas para o escalonamento de requisições em RMEP. CBase [Kotla and Dahlin 2004] utiliza um grafo para manter controle das dependências entre as requisições, em [Mendizabal et al. 2017] mapas de bits anotam informação de dependência entre comandos, enquanto lotes de requisições são utilizados para aumentar o taxa de ocupação nas threads executoras. Outras técnicas separam as requisições em classes de conflito e definem o relacionamento entre as classes [Alchieri et al. 2018]. Uma das dificuldades ao desenvolver uma nova técnica, porém, é medir o potencial ganho em paralelismo que a técnica pode extrair e como compará-la com técnicas já existentes, sem que haja a necessidade de implementá-la em um sistema completo.

Nesse trabalho é descrito um simulador de execução de requisições em RMEP, para analisar o paralelismo obtido por algoritmos de escalonamento, abstraindo demais custos de uma implementação real. Durante a simulação, requisições são geradas e enviadas para o algoritmo de escalonamento, que registra dados da simulação para posterior análise. As etapas da execução são modularizadas de forma que é possível editar aspectos pontuais da simulação para representar diferentes ambientes e comportamentos. Através dos dados levantados, é possível medir o potencial paralelismo obtido por políticas de escalonamento, observar como diferentes parâmetros afetam o desempenho, e comparar técnicas, antes de implementá-las em um sistema real. 


\section{Funcionamento do simulador}

O uso do simulador passa pelas configurações iniciais, geração de requisições, execução e saída de dados. A Figura 1 mostra a arquitetura do simulador.

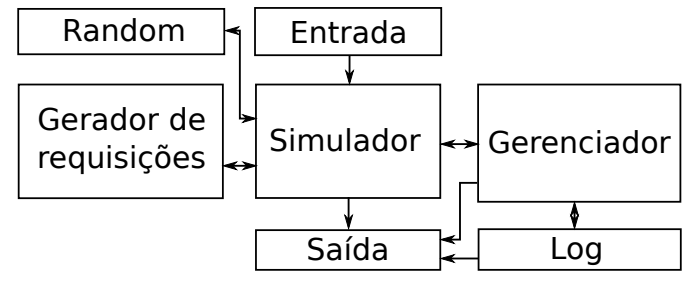

Figura 1. Arquitetura do simulador

As configurações do simulador, como número de chaves no sistema, quantidade e distribuição de requisições, são especificadas em um arquivo de entrada. É no arquivo de configuração que são definidos comportamentos que podem variar entre diferentes simulações, visando a análise da execução sob diferentes parâmetros.

Requisições podem ser geradas de maneira aleatória ou importadas de um arquivo externo. Existem dois tipos de requisições, requisições que acessam uma única chave, e requisições que acessam múltiplas chaves. Caso sejam importadas de um arquivo externo, o caminho de um arquivo definindo uma lista de requisições é esperado. Se for necessário gerar requisições aleatórias, o simulador chama o módulo Random, como demonstrado na Figura 1, para buscar geradores de números pseudo-aleatórios que satisfaçam a distribuição desejada.

Ao optar por gerar requisições aleatórias, em requisições de chave única é possível especificar a quantidade de requisições e a distribuição a ser utilizada durante a geração. Em requisições que acessam múltiplas chaves, é preciso definir a quantidade de requisições, o número mínimo e máximo de chaves envolvidas, e a distribuição utilizada para escolher o tamanho e as chaves envolvidas nas requisições. Atualmente estão implementadas as distribuições fixa, uniforme e binomial.

Durante a execução da simulação é assumido que todas as requisições tenham o mesmo tempo de execução, definido como uma unidade de tempo. Seguindo a arquitetura demonstrada na Figura 1, o Simulador interage com o módulo Gerenciador, que deve ser implementado estendendo uma classe de mesmo nome. É preciso que a nova classe implemente o método execute_requests, que deve realizar o escalonamento das execuções. Durante o escalonamento, é possível interagir com o Log para administrar o estado de cada thread simulada.

Após a execução, o simulador escreve em um arquivo informações sobre as threads simuladas. São apresentados o instante de tempo em que as threads terminaram de executar a última requisição, a quantidade de tempo em que estiveram ociosas e a porcentagem total que esse tempo representa. Essas informações são obtidas através do Log, utilizado durante o escalonamento. Além das informações coletadas pelo Log, o Gerenciador pode, através do método export_data, escrever dados adicionais. As requisições utilizadas durante a execução podem ser exportadas e reutilizadas em simulações futuras. 


\section{Análise de uso: comparando técnicas}

Para mostrar o simulador em funcionamento, foram implementadas variações do Gerenciador para duas técnicas de escalonamento: CBase e corte em grafo. Dada a estrutura modular da ferramenta, outras estratégias de escalonamento podem ser facilmente incorporadas ao simulador futuramente.

O CBase [Kotla and Dahlin 2004] cria um grafo de dependências, em que vértices são requisições e existe uma aresta $(x, y)$ se, e somente se, a execução de $x$ antecede $y$. Threads acessam o grafo e executam as requisições representadas por vértices fonte, e depois disso atualizam o grafo, removendo o vértice. Esse algoritmo é considerado ótimo em exploração de paralelismo, porém implementações reais apresentam um grande custo devido ao acesso concorrente ao grafo e à detecção de conflitos [Mendizabal et al. 2017].

O corte mínimo em grafos é uma técnica de balanceamento de carga (e.g. [Hendrickson and Kolda 2000]). O grafo aqui é modelado de forma que os vértices são chaves, seu peso é a quantidade de vezes que foram acessadas, e uma aresta $(a, b, x)$ diz que as chaves $a$ e $b$ foram acessados por uma mesma requisição $x$ vezes, onde $x>0$. $\mathrm{O}$ corte resulta em conjuntos disjuntos de chaves, chamados de partições, cada uma atribuída a uma thread. A execução de requisições é feita em paralelo por todas as threads, cada uma executando as requisições nas chaves em suas partições. Caso uma requisição acesse chaves que estão em partições diferentes, as threads que possuem as chaves acessadas sincronizam a execução do comando, onde apenas uma executa o comando, e as demais ficam bloqueadas. O número de partições é definido como o mesmo número de threads, e inicialmente as chaves são distribuídas pelas partições utilizando Round-Robin. A biblioteca METIS [Karypis and Kumar 1998] foi utilizada para realizar o corte mínimo.

As simulações foram configuradas com 1.000 chaves e 1.000 .000 de requisições, sendo que $75 \%$ das requisições acessam uma única chave e as demais acessam múltiplas chaves. Para gerar requisições de um único valor, foi utilizada a distribuição binomial na escolha da chave; para requisições que acessam múltiplas chaves, o número de chaves pode variar de 2 a 8 , de acordo com uma distribuição binomial, e a escolha das chaves segue uma distribuição uniforme. Duas situações do corte em grafos foram exploradas, uma com reparticionamento a cada 250.000 requisições, e outra com reparticionamento a cada 100.000 requisições. Foram simulados cenários com 2, 4, 8 e 16 threads.

A Figura 2(a) mostra o gráfico do tempo necessário para a execução das requisições, enquanto a Figura 2(b) exibe o tempo médio em que as threads ficam ociosas, indicados em unidades de tempo (u.t.). A ociosidade é computada pela thread a cada instante em que aguarda pela sincronização com outra(s) thread(s). Apesar do alto custo de implementação, o CBase é ideal em termos de exploração do paralelismo, o que causa um baixo tempo de execução e nenhuma ociosidade. Nos escalonamentos utilizando corte mínimo, o tempo em que as threads permanecem ociosas é maior, devido à necessidade de sincronizações. Isso reflete no tempo total de execução, maior do que o obtido com o CBase. É possível perceber como a frequência de reparticionamento pouco afetou o tempo de execução ou a ociosidade, e que uma frequência maior pode, por vezes, deteriorar o desempenho. O simulador tem como objetivo levantar informações sobre número de sincronizações, ociosidade das threads e tempo de execução total de maneira simples, para que sejam considerados durante a concepção de novos algoritmos ou em comparações entre diferentes técnicas e configurações. 


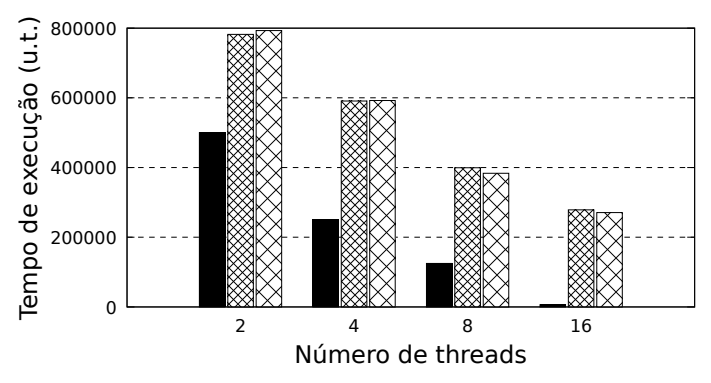

(a) Tempo total de execução.

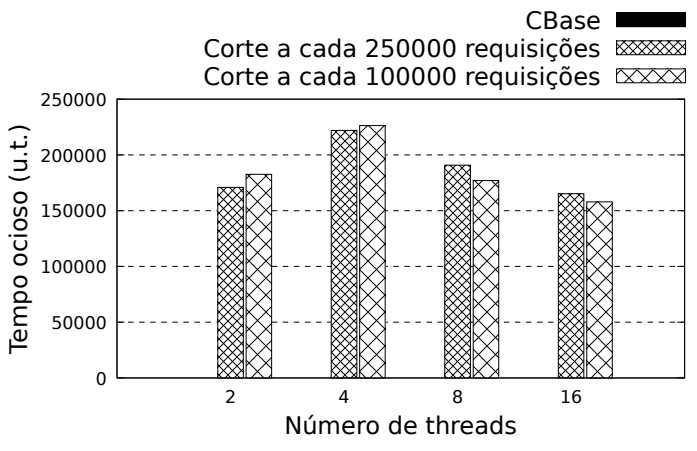

(b) Média do tempo ocioso entre as threads.

Figura 2. Gráficos obtidos a partir das informações de saída do simulador.

\section{Considerações finais}

Com a introdução de Replicação Máquina de Estados Paralela, é necessário que sejam exploradas técnicas de escalonamento de requisições que consigam extrair melhor paralelismo do modelo. É apresentando, então, um simulador que abstrai custos de uma implementação real, para que durante o concebimento de um novo algoritmo seja possível analisar o potencial paralelismo da técnica sob diferentes configurações. Podese, também, comparar algoritmos existentes, como um instrumento de análise.

\section{Agradecimentos}

O presente trabalho foi realizado com o apoio do Conselho Nacional de Desenvolvimento Científico e Tecnológico - CNPq - Brasil.

\section{Referências}

Alchieri, E., Dotti, F., Marandi, P., Mendizabal, O., and Pedone, F. (2018). Boosting state machine replication with concurrent execution. In 2018 Eighth Latin-American Symposium on Dependable Computing (LADC).

Hendrickson, B. and Kolda, T. G. (2000). Graph partitioning models for parallel computing. Parallel computing, 26(12):1519-1534.

Karypis, G. and Kumar, V. (1998). A fast and high quality multilevel scheme for partitioning irregular graphs. SIAM Journal on scientific Computing, 20(1):359-392.

Kotla, R. and Dahlin, M. (2004). High throughput byzantine fault tolerance. In International Conference on Dependable Systems and Networks, 2004.

Lamport, L. (1978). Time, clocks, and the ordering of events in a distributed system. Communications of the ACM.

Mendizabal, O. M., De Moura, R. S., Dotti, F. L., and Pedone, F. (2017). Efficient and deterministic scheduling for parallel state machine replication. In 2017 IEEE International Parallel and Distributed Processing Symposium (IPDPS).

Schneider, F. B. (1990). Implementing fault-tolerant services using the state machine approach: A tutorial. ACM Computing Surveys (CSUR), 22(4):299-319. 